\title{
REFERENCE
}

\section{Magnetic Recording of Acoustical Data on Audiofrequency Tape}

U.S. PARTMENT OF OMMERCE National Bureau ac 100 us753 0.718 1972 
The National Bureau of Standards ${ }^{1}$ was established by an act of Congress March 3, 1901. The Bureau's overall goal is to strengthen and advance the Nation's science and technology and facilitate their effective application for public benefit. To this end, the Bureau conducts research and provides: (1) a basis for the Nation's physical measurement system, (2) scientific and technological services for industry and government, (3) a technical basis for equity in trade, and (4) technical services to promote public safety. The Bureau consists of the Institute for Basic Standards, the Institute for Materials Research, the Institute for Applied Technology, the Center for Computer Sciences and Technology, and the Office for Information Programs.

THE INSTITUTE FOR BASIC STANDARDS provides the central basis within the United States of a complete and consistent system of physical measurement; coordinates that system with measurement systems of other nations; and furnishes essential services leading to accurate and uniform physical measurements throughout the Nation's scientific community, industry, and commerce. The Institute consists of a Center for Radiation Research, an Office of Measurement Services and the following divisions:

Applied Mathematics-Electricity-Heat-Mechanics-Optical Physics-Linac Radiation ${ }^{2}$ - Nuclear Radiation ${ }^{2}$ - Applied Radiation ${ }^{2}$-Quantum Electronics ${ }^{3}$ Electromagnetics ${ }^{3}$-Time and Frequency ${ }^{3}$ - Laboratory Astrophysics ${ }^{3}$ - Cryogenics ${ }^{3}$.

THE INSTITUTE FOR MATERIALS RESEARCH conducts materials research leading to improved methods of measurement, standards, and data on the properties of well-characterized materials needed by industry, commerce, educational institutions, and Government; provides advisory and research services to other Government agencies; and develops, produces, and distributes standard reference materials. The Institute consists of the Office of Standard Reference Materials and the following divisions:

Analytical Chemistry-Polymers-Metallurgy-Inorganic Materials-Reactor Radiation-Physical Chemistry.

THE INSTITUTE FOR APPLIED TECHNOLOGY provides technical services to promote the use of available technology and to facilitate technological innovation in industry and Government; cooperates with public and private organizations leading to the development of technological standards (including mandatory safety standards), codes and methods of test; and provides technical advice and services to Government agencies upon request. The Institute also monitors NBS engineering standards activities and provides liaison between NBS and national and international engineering standards bodies. The Institute consists of the following divisions and offices:

Engineering Standards Services-Weights and Measures-Invention and Innovation-Product Evaluation Technology-Building Research-Electronic Technology-Technical Analysis-Measurement Engineering-Office of Fire Programs.

THE CENTER FOR COMPUTER SCIENCES AND TECHNOLOGY conducts research and provides technical services designed to aid Government agencies in improving cost effectiveness in the conduct of their programs through the selection, acquisition, and effective utilization of automatic data processing equipment; and serves as the principal focus within the executive branch for the development of Federal standards for automatic data processing equipment, techniques, and computer languages. The Center consists of the following offices and divisions:

Information Processing Standards-Computer Information-Computer Services - Systems Development-Information Processing Technology.

THE OFFICE FOR INFORMATION PROGRAMS promotes optimum dissemination and accessibility of scientific information generated within NBS and other agencies of the Federal Government; promotes the development of the National Standard Reference Data System and a system of information analysis centers dealing with the broader aspects of the National Measurement System; provides appropriate services to ensure that the NBS staff has optimum accessibility to the scientific information of the world, and directs the public information activities of the Bureau. The Office consists of the following organizational units:

Office of Standard Reference Data-Office of Technical Information and Publications-Library-Office of International Relations.

\footnotetext{
1 Headquarters and Laboratories at Gaithersburg, Maryland, unless otherwise noted; mailing address Washington, D.C. 20234.

2 Part of the Center for Radiation Research.

Located at Boulder, Colorado 80302.
} 


\section{Magnetic Recording \\ of Acoustic Data \\ on Audiofrequency Tape}

Edwin D. Burnett, Edith L. R. Corliss and

Raymond D. Berendt

Mechanics Division

Institute for Basic Standards

National Bureau of Standards

Washington, D.C. 20234

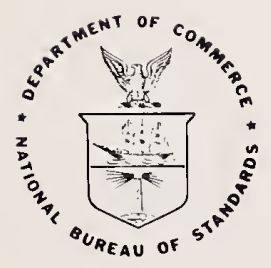

U.S. DEPARTMENT OF COMMERCE, Peter G. Peterson, Secretary NATIONAL BUREAU OF STANDARDS, Lewis M. Branscomb, Director,

Issued April 1972 
National Bureau of Standards Technical Note 718

Nat. Bur. Stand. (U.S.), Tech. Note 718,29 pages (Apr. 1972)

CODEN: NBTNAE

Issued April 1972

For sale by the Superintendent of Documents, U.S. Government Printing Office Washintgon, D.C. 20402 (Order by SD Catalog No. C 13.46:718). Price 40 cents. 
TABLE OF CONTENTS

PAGE

1. INTRODUCTION . . . . . . . . . . . . . . . . . . . . 1

2. THE RECORDING MEDIUM . . . . . . . . . . . . . . . . . 2

3. TAPE FORMAT . . . . . . . . . . . . . . . . . 6

4. TAPE SPEED . . . . . . . . . . . . . . . . . . . 7

5. RECORDING LEVELS . . . . . . . . . . . . . . . . . 10

6. RECORDER ADJUSTMENTS . . . . . . . . . . . . . . . . 11

6.1. Head Azimuth Alignment . . . . . . . . . . . . 12

6.2. Bias Adjustment . . . . . . . . . . . . . . 13

6.3. Noise Balance . . . . . . . . . . . . . . . 14

6.4. Miscellaneous Good Housekeeping Procedures . . . . . . 15

7. TAPE RECORDERS FOR RESEARCH PURPOSES . . . . . . . . . . . 16

8. RERECORDING . . . . . . . . . . . . . . . . . 19

9. TAPE EDITING . . . . . . . . . . . . . . . . . . 20

10. MICROPHONE TECHNIQUES . . . . . . . . . . . . . . . . . 21

11. REFERENCES . . . . . . . . . . . . . . . . . . 27

12. GLOSSARY • . . . . . . . . . . . . . . . . . . 28 

Edwin D. Bumett, Edith L. R. Corliss, and Raymond D. Berendt

This Technical Note discusses the application of magnetic tape recording to storage and analysis of data obtained in the course of acoustic measurements. In general, the most suitable machines are those designed primarily for recording studios. Advantages in signal-to-noise ratios and extended linear range can be realized by careful choice of recording medium, adjustment of the equipment to optimum performance, and proper care in monitoring the recording process. General instructions for realizing these advantages are given. Particular emphasis is given to the procedures for making well-calibrated recordings in the field, for analysis of noise problems.

Key words: Acoustic measurements; calibrated tape recordings; data acquisition;. magnetic recording; psychoacoustic data; recordings in the field; tape recording.

\section{INTRODUCTION}

Audio-type tape machines, ordinarily used for recording music, are widely used for instrumentation applications. For example, in studies of community noise, signals are recorded in the field on tape to be analyzed 1ater. In psychoacoustic experiments, where the same stimuli are to be presented to a number of human subjects, the tape storage medium makes the experiments feasible.

The machines used are often admirably suited for technical work of this nature, even though the original purpose for which they were designed is more esthetic than quantitative. However, there are limitations in performance that are not obvious to everyone using these recorders. We wish to point out these limitations, and to describe methods for obtaining optimum results. Some of the material we will present is wide$1 y$, perhaps universally, recognized in the commercial studio recording industry, but seems little known to those whose specialty is not tape recording.

The information given here supplements the detailed instructions given in the manuals supplied by manufacturers of recording equipment. 
The procedures given in the manuals apply to the specific equipment provided by the manufacturer. The suggestions presented here are general techniques for improving the adjustment of recorders, maintaining close control of the level of the signals on the tape, and maintaining the best adjustments for dynamic range and bandwidth.

An extended discussion of the levels and equalization characteristics used in recording can be found in J. G. McKnight's paper, "Absolute Flux and Frequency Response Characteristics in Magnetic Recording" [1]. 'I We suggest that this paper be read by anyone concerned with magnetic recording.

Although general principles for maintaining the quality of magnetic tape recordings are common to all recorders, the material to be presented here is not intended to apply in all respects to analog instrumentation recorders which may be used for recording in a frequency band other than the audiofrequency range, and which may often use different types of tape, different equalization, tape speeds, and track format.

The premise taken throughout is that the costs of the magnetic recorders and recording tape are negligible as compared to the rest of the experiment or data-taking process, and that any compromise that economizes on tape costs against results is necessarily a poor compromise.

\section{THE RECORDING MEDIUM}

For serious measurements of audiofrequency phenomena or for psychoacoustic work, one should make use of the best tapes available. In general, many of the design problems in the tape recording machines themselves are more readily solved than are the difficulties inherent in the tape medium. The limitations of the tape itself will be the major constraints on linearity and signal-to-noise ratio, if the recording is made on a good tape machine.

There have been significant improvements in tape in the last few years. "Low-noise tapes" are now made by several manufacturers. These low-noise tapes will permit a five-dB greater dynamic range ${ }^{2}$ to be recorded than will the usual commercial tapes. A special tape is also 1 Figures in brackets indicate the literature references on page 27. 2 Refer to Glossary for definitions of underlined terms. 
available that is claimed to have a dynamic range three $\mathrm{dB}$ better than even the low-noise tapes. It is intended for use in making tape masters. It uses nominally the same conditions for equalization and bias as does the low-noise tape made by the same manufacturer, so that it is interchangeable on a tape recorder set up for low-noise tapes of corresponding origin with a minimum of further adjustment.

Also recently available is a "low-noise, low-print" tape, which has the same nominal characteristics as "low-noise" tape, except for five dB less print-through. This is an excellent tape for speech, where there may be silent portions where print-through is particularly obvious. This would also be our tape of choice where long-term storage is contemplated. With a two-channel tape recorder in good adjustment, a signal-tonoise ratio of $60 \mathrm{~dB}$, with an input signal level of $+6 \mathrm{vu}$, should be available with low-noise tapes. On a single-channel full-width recording, the corresponding signal-to-noise ratio should be $65 \mathrm{~dB}$. It should however, be pointed out that these figures are for the ratio between a particular level of a single frequency recorded on the tape, and the noise in a quiet, unrecorded portion of the tape. Actually, accompanying each signal recorded on the tape there is another noise that is difficult to evaluate, called the "modulation noise". This noise is a fixed percentage of the signal level, usually approaching one percent of the signal amplitude (i.e., about $40 \mathrm{~dB}$ below the signal level). This noise is well above the level of the residual noise on a tape that has been passed through the recording process with no signal applied.

The dynamic characteristics of overload at the high end of the recording range are nearly alike for many American-made tapes. At the "0 vu" level on American tape recorders, corresponding to a magnetic flux of $210 \mathrm{nWb} / \mathrm{m}$, the distortion is nominally one percent at $400 \mathrm{~Hz}$. With most modern tapes, this nominal figure is a bit high; typically, the distortion is about 0.7 percent. At a level six $\mathrm{dB}$ above $0 \mathrm{vu}$, the nominal distortion at $400 \mathrm{~Hz}$ is given as three percent (but usually observed. to be about two percent). Saturation at low frequencies (those frequencies having recorded wavelengths of more than $10 \mathrm{mils}$ [.25 mm]) occurs for an Input leve1 about $14 \mathrm{~dB}$ above the $210 \mathrm{nWb} / \mathrm{m}$ reference flux level. For this input, the output at saturation is $12 \mathrm{~dB}$ above 0 vu output. 
There are tapes that differ somewhat in their properties from the general features given above. For example, the mastering tape referred to earlier remains linear to levels about three dB higher than the above figures. In general, tapes designated as "double-play" and "triple-play" will have inferior overload characteristics and require different bias and equalization adjustments because of the thinner oxide coatings. High-output tape differs in its coating, also, and requires special adjustments.

We checked the characteristics of three types of tape: 1ow-noise tape, master tape, and triple-play tape. The results are shown in Table 1, for 15 ips (inches per second) and Table 2, for 7-1/2 ips. These data were obtained with the high-frequency record equalization disconnected, so that the variation of sensitivity with frequency can be seen. The significance of the changes with tape speed and bias condition will be discussed later. Here, we shall discuss the characteristics at 15 ips and with peak bias.

The saturation output at $1 \mathrm{kHz}$ may be considered as being representative of all frequencies below $1 \mathrm{kHz}$. In recording, a pre-emphasis of 3 $\mathrm{dB}$ at $50 \mathrm{~Hz}$ is applied, with a corresponding de-emphasis on playback. This reduces the apparent saturation level at very low frequencies by the amount of the playback de-emphasis. All tapes are affected equally by this so we need not consider frequencies below $1 \mathrm{kHz}$ in comparison studies.

The most important figures are the saturation output and the noise level, as these determine the absolute dynamic range that can be recorded. Notice that for low-noise tape the saturation level is nearly independent of frequency. This is a desirable situation for instrumentation applications. At low frequencies, the mastering tape does saturate at a level $2.5 \mathrm{~dB}$ higher than does low-noise tape, which is essentially as is claimed for it. At high frequencies, the saturation level decreases to slightly below that of low-noise tape. The noise level was $2 \mathrm{~dB}$ less for the low-noise tape than for the master tape, so the dynamic range available with these particular lots of tape was essentially the same for the two types. The master tape met its noise specifications; 
the low-noise tape was $2 \mathrm{~dB}$ quieter than its specifications called for. This bonus cannot be assumed for all batches of the tape.

Triple-play tape has a low-frequency saturation level which is 6.5 $\mathrm{dB}$ less than that of low-noise tape. Its noise is $5 \mathrm{~dB}$ higher, so the overall dynamic range is $11.5 \mathrm{~dB}$ less than for low-noise tape. Its high frequency saturation level increases to that of low-noise tape, but for the spectral distributions found in most signals this does not justify its use for audio instrumentation applications.

Some types of instrumentation tape will perform very well in audio applications and, depending on the tape, will offer a somewhat more uniform output. For applications where this is of paramount importance, an appropriate instrumentation tape should be considered. Otherwise, lownoise tape is usually superior for audio applications. Some instrumentation tapes are designed for very short wavelength recording, and must be avoided for audio range work as their dynamic range is limited.

In Ref. [2] a tabulated summary of the physical and magnetic properties of a number of tapes of European and American origin is presented.

There is a tendency for tapes of European origin to saturate at a higher level than American-made tapes. The tape noise is also higher, so the overall signal-to-noise ratio is not necessarily better. These relations do not apply to every tape type; the characteristics of any type under consideration should be examined individually.

In an effort to extend the dynamic range of tape recordings, some tape recorders provide a "Iinearity control". This control actually introduces a controlled amount of odd-order harmonic distortion, opposite in phase to the odd-order harmonic distortion that normally appears at high levels in the recording tape. This can produce a material decrease of distortion at levels where saturation distortion is incipient, but obviously it cannot permit linear recording beyond the absolute limit of saturation on the tape. Unfortunately, the phase characteristics of the recording process may change at high frequencies, and the linearization process may, in fact, increase the distortion at high frequencies. This pitfall should be kept in mind when the signals to be recorded have a large content of high-frequency components. 
Most types of magnetic coatings for tape recording are available with a choice of either acetate or polyester backings. Special-purpose tapes are usually made with polyester backings. Under almost any conditions, the polyester backings are to be preferred. Acetate backings are unreliable for repeated use or long-term storage. They require careful handling, and must be protected from large changes of humidity; even then they are liable to warping - usually this happens to the tape you need the most.

The standard-weight polyester backing is $1.5 \mathrm{mils}(38 \mu \mathrm{m})$ thick. It is tough and durable. Polyester backings one mil $(25 \mu \mathrm{m})$ thick have an adequate mechanical strength for many purposes, and permit a playing time about 50 percent longer than that of the 1.5-mil stock, but the problems of print-through are correspondingly enhanced. However, if the tapes are not likely to be exposed to temperatures in excess of normal room temperature, one-mil tape should prove usable if the extra storage capacity is really needed.

The thin backings of double- and triple-play tapes are not suited to quantitative use. They stretch readily, and print-through problems are severe.

A tape formulation using chromium dioxide instead of the presentlyused ferrous oxide has been developed. It offers a superior signal-tonoise ratio and frequency range. It is not yet available except in limited amounts for cassette tapes. It will have quite different requirements for drive and bias, but these requirements should be within the operating range of most professional machines.

\section{TAPE FORMAT}

For each doubling of the number of tracks recorded on tape of a given width, there is a sacrifice of about five $d B$ in the signal-to-noise ratio. The four-track format was designed for home use, and is not suitable for either quantitative measurements or psychoacoustic work. Not only is the tape noise an increased constraint on performance, but the narrow track width makes transverse orientation with respect to the head critical. Any tape weave produces significant variations in the playback amplitude. 
Two- and four-track formats are not compatible. The upper track, or left-hand channel when recording stereo, of a two-track tape may be playable on a four-track machine if the recording on the track extends to the outer edge of the tape (but there is no standard requiring this). Playing back the upper track of a four-track tape on a two-track machine will yield a signal-to-noise ratio degraded by five $\mathrm{dB}$ or more as compared to playback over a corresponding four-track head. The lower track of a twotrack recording and the number three track of a four-track recording occupy spaces that barely overlap.

Unless recordings are to be made in stereo, we would recommend using a full-track recorder wherever one is available. If all work must be done with a two-track machine, an increase of signa1-to-noise ratio of three $\mathrm{dB}$ can be realized by making simultaneous in-phase recordings on both channels. The two inputs are driven in paralle1. On playback, the outputs of the two channels cannot be coupled directly togethër, but both the signals may be combined via isolation pads. To avoid phasing problems, the heads must be in precise alignment.

It is possible to extend the usable dynamic range of a tape recording even further by the stratagem of making simultaneous recordings on both tracks of a two-channel recorder, setting the input attenuator of one channel so that the recording level is of the order of $15 \mathrm{~dB}$ above the other. On playback, one can select the appropriate channel with the necessary complementary attenuation.

The signal-to-noise ratio of tape recordings can be increased by the use of compression-expansion devices which process different frequency regions independently [3]. If such a device is used for instrumentation purposes, very careful calibration procedures must be used, preferably using both sinusoids and gated sinusoids for checking levels.

\section{TAPE SPEED}

Upon inspection of the manufacturers' literature, it might be easy to conclude that the frequency range and signal-to-noise ratios of recordings are about as good at a tape speed of $7-1 / 2$ ips $(19.05 \mathrm{~cm} / \mathrm{s})$ as they are for the tape speed of $15 \mathrm{ips}(38.1 \mathrm{~cm} / \mathrm{s})$. There seems to be a 
widespread assumption that the qualities of recordings made at the lower speed are essentially equivalent to those obtained at the higher speed. Lost in the specifications is the fact that pre-emphasis is required at the high-frequency end of the range when the lower tape speed is used, in order to achieve uniform response over the specified frequency range. As a result, on most kinds of recording tape, signals with appreciable energy in the vicinity of ten $\mathrm{kHz}$ will saturate the tape when recorded at an input level of two $d B$ referred to 0 vu [4]. At a speed of 7-1/2 ips, a commonly required characteristic for pre-emphasis is the imposition of a six-dB per octave slope, with the transition frequency set at $2800 \mathrm{~Hz}$. On the other hand, when a speed of 15 ips is used, the total pre-emphasis required at $10000 \mathrm{~Hz}$ is three $\mathrm{dB}$ or less [1].

Because of the reduced wavelength at slower recording speeds, fluctuations in recorded level are more severe in the high-frequency range at slow speeds. Figure 1 shows the envelope that results from recording a steady-state signal of $10000 \mathrm{~Hz}$ at $7-1 / 2$ and 15 ips. These recordings were made under as nearly optimum conditions as could be obtained, and indicate the best results that may be expected.

If the greater fluctuations in level can be tolerated, recordings adequate for some purposes can be made at 7-1/2 ips, but the limitations on dynamic range at high frequencies must be taken into account. The distribution of peak energy in the signal as a function of frequency must be held below the complement of the pre-emphasis used in recording. Thus, some degree of sacrifice of overall signal-to-noise ratio is required. Even with this compromise, the recorder must be maintained in optimum condition with respect to mechanical alignment, bias, and equalization, to preserve the dynamic and frequency range at 7-1/2 ips.

We would infer from data on the peak energy distributions of speech sounds [5] that the recording of speech at 7-1/2 ips for the purpose of studying speech sounds is a marginal proposition. Notice that we are not discussing recording for broadcast or home use, where the meaning of the speech is paramount, and the occasional saturation of a short-duration peak is not likely to be noticed. 


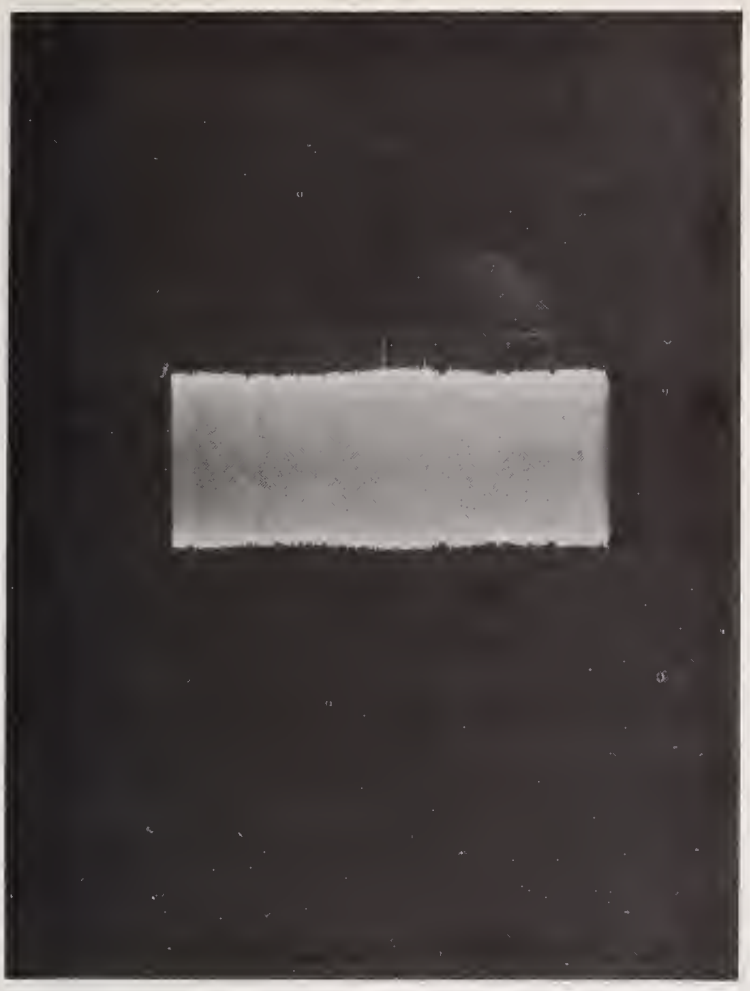

(A)

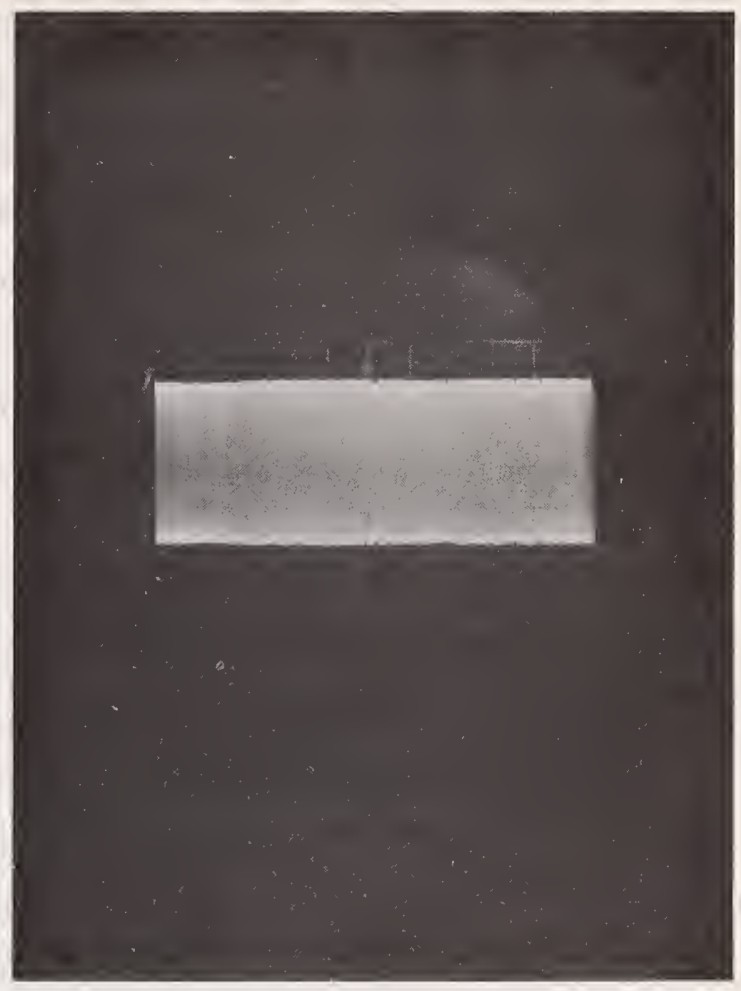

(B)

Figure 1. (A) Tape speed of 7.5 ips. Output of low-noise tape with an input of $10 \mathrm{kHz} .20$ milliseconds per division.

(B) Tape speed of 15 ips. Other conditions are the same as for (A).

Thus, although most tape recorders have the possibility of recording at 7-1/2 ips, this speed should not be considered the most generally useful speed. At a transport speed of 15 ips, most limitations in the highfrequency region are displaced upward by an octave and alignment errors become less critical. Because of the very slight demands for equalization, dynamic range is at its widest over a much larger range of frequencies.

For the purposes of recording quantitative information in the audiofrequency range, speeds of $3-3 / 4$ ips and below should be avoided.

On a 10-1/2-inch reel of tape at 15 ips, 48 minutes of usable recording can be stored using one-mil tape. It is usually more advantageous to spend some effort in organizing the taking of data to meet this limitation on the duration of uninterrupted recording than to spend at least an 
equal amount of time adjusting a recorder to function within the limitations imposed by the 7-1/2 ips speed.

Situations do arise in which the recording time limitation must be considered, especially if the tape machine available can handle only a seven-inch reel. This size limits the maximum recording time to 24 minutes at 15 ips using tape with one-mil backing. If this time must be exceeded in a continuous run, a choice must be made between using a thinner tape or a tape speed of 7-1/2 ips. Usually, the lower tape speed is the preferable option.

One exception, which must be almost unique, would be the recording of songbirds. Triple-play tape does have excellent high-frequency characteristics. For bird calls, the response should extend to the near ultrasonic. The dynamic range is not a handicap because it is already limited by the background noise of the environment. The input level must be reduced to avoid overload, and the bias and equalization readjusted for this tape. The recorded signal should be copied to a thick base tape before studying or long-term storage of the material.

\section{RECORDING LEVELS}

The most common flaw that we find in tape recordings, when some error has been made, is that the recordings have been made at too high an input level. There seems to be the very human tendency to worry whether the signal is actually getting onto the tape, and so it is pounded into the tape. As a result, the signal is on the tape, for sure, and so are a lot of distortion products generated by overloading the tape.

This problem is not mitigated by the type of recording indicator used on most American machines. The Vu meter has very sluggish ballistics, and does not begin to follow the peak levels actually going into the recording head. Most meters are set so that the 0-vu indication is $12 \mathrm{~dB}$ below the saturation output limit of the tape for steady-state signals. This allowance is adequate for signals with peaking factors of ten $\mathrm{dB}$ or less. A sinusoidal signal has a factor of three $\mathrm{dB}$ between the absolute peak and rms levels. (The peaking factor for fluctuating signals is usually defined as the ratio by which the peak amplitudes of the signal exceed, for some fraction of the time, the long-term rms level of 
the signal. A figure often used is the peak level exceeded about ten percent of the time.) Speech has a peaking factor that may range from 15 to $20 \mathrm{~dB}$. Thus, the actual allowance to be made for peaks is not immediately obvious, and varies with the signal characteristics. Clearly, for the brief consonant sounds that occur frequently in the English Ianguage, the allowance between 0 vu and saturation is not adequate, and for accurate representation of speech sounds, the long-time level at which speech is recorded should be set at less than 0 vu.

Our experience with recording speech in an anechoic chamber, using a tape speed of 15 ips, has been that even when we had a tape recorder in very good adjustment, we had to hold the level of recording so that peaks read on the meter were always below $-7 \mathrm{vu}$, in order to avoid audible distortion. The European type of peak program meter (such as the one designed by the $\mathrm{BBC}$ ) yields indications better correlated with those properties of the signal that must be monitored while recording. The American VU meter is easy to read; unfortunately it does not indicate accurately any necessary feature when fluctuating signals are applied.

For critical recording, where accurate knowledge of the peaks must be preserved, an oscilloscope can be used to monitor the recording levels. Where separate outputs are available to permit monitoring the signals at the recording and playback heads, a dual-channel oscilloscope makes it possible to compare the instantaneous waveforms of the input and recorded signals. Magic-eye indicators are really a simplified form of oscilloscope, and though they are cheap, and appear often on inexpensive machines intended for home use, they are actually capable of a better indication of peak recording levels than is the VU meter. Unhappily, they are often slowed in their response by additional circuits, in order to approximate the ballistics of a VU meter.

\section{RECORDER ADJUSTMENTS}

Even the best tape recorders require careful adjustment to produce optimum results. (Poor machines often permit no adjustments.) Bias must be set for the type of tape being used; the adjustment depends upon both the composition and the thickness of the magnetic coating on the 
tape. If the user is really trying for the best possible performance, adjustments may be made for each lot of tape. The instructions prescribed by the manufacturers of the machines are usually adequate, so we are not attempting to give a complete alignment procedure. We will describe several techniques which are not always given in instruction manuals, or may be at variance with the manuals, but which will usually give superior results.

\subsection{Head Azimuth Alignment}

On machines with separate recording and playback heads, the first part of the procedure is to align the playback head by means of a standard alignment tape. The next step is the alignment of recording and playback azimuths together.

On a multichannel machine, this procedure is facilitated and made more accurate by using a Lissajous pattern between the channel outputs. Connect the outputs of each channel of a two-channel recorder to the $X$ and $Y$ inputs, respectively, of an oscilloscope.

For a single channel, the azimuth of the playback head is adjusted to produce maximum amplitude for playback from the high-frequency signals on the alignment tape. On a two-channel recorder, this adjustment is made for one channel in order to give an approximate alignment, and then the playback head assembly is adjusted in azimuth until the outputs of the two channels, as monitored on the $\mathrm{X}$ and $\mathrm{Y}$ channels of an oscilloscope, produce the best straight line Lissajous pattern at all frequencies. Where more than two channels are used on the machine, the most sensitive adjustment is to line up the outputs of the extreme heads.

The quickest way of setting the best straight line is to use the volce portions of the standard alignment tape. This setting can then be checked against the playback of discrete frequencies. The phase delay in each playback head is now identical. Since the alignment tape has been recorded with the signals accurately perpendicular to the direction of run of the tape, the two playback heads have now been set into proper alignment. If the maximum output at high frequencies for playback from the alignment tape does not coincide with the best line pattern in the 
Lissajous figure, the heads were poorly assembled in the first place, and only a compromise adjustment can be made. At worst, the phase difference should not be more than $90^{\circ}$ at any frequency. For a phase difference of $90^{\circ}$, the Lissajous pattern is spread into a circle.

It remains now to line up the record heads. On a single-channel machine, the record head is set simply to give the maximum playback of high-frequency signals from the previously aligned playback head. For a multichannel machine, a more sensitive process is to use a white noise at the input to both recording channels, and to adjust the azimuth of the recording heads to produce the best straight line in the Lissajous pattern of the signal from the playback heads.

(If you find yourself with a tape recorder whose head alignment you suspect to be incorrect, and are without an alignment tape, it is at least theoretically possible to align the heads by following the basic procedure used to generate alignment tapes. The first adjustment is to align the record and playback heads to produce maximum output at high frequencies. The tape recording made for this purpose is then removed from the machine and reversed completely, so that the backing is toward the heads, and the playback amplitudes are checked. Of course the output will be considerably lower, but what you are looking for is the dependence of playback amplitude upon frequency. The recording and playback heads will have the best alignment possible when the playback of high frequencies shows the least droop after reversal of the tape.)

\subsection{Bias Adjustment}

Best results are obtained for usual circumstances by overbiasing the tape about one $d B$. That is, while recording a low frequency, e.g. 400 $\mathrm{Hz}$, the bias level is raised until the output is at a maximum on playback, and then raised still further until the output level droops to about one $d B$ below the maximum. This causes the magnetization to penetrate further into the tape, and reduces modulation noise [6].

An equally important advantage to be derived from overbiasing is the possibility of keeping above the bias level at which excess distortion sets in. This can be seen from the way distortion depends upon bias. As the bias is increased beyond the setting for maximum output, the 
distortion remains essentially constant. As the bias is decreased below the setting which gives maximum output, the distortion rises markedly as the bias level declines. Thus, if the bias is set for maximum output, any factor that may tend to decrease the bias, such as low line voltage, will have the effect of raising the amount of distortion. If the machine has been set to overbias in the first place, the only result of the line voltage dropping is a slight change in level. Although the precisé amount of overbiasing will in general depend upon the tape, as a rule an increase of about one $d B$ in the bias level beyond that needed for peak output will correspond closely to the overbias for a one-dB signal reduction.

With most tapes, it may be necessary to follow the overbiasing adjustment with a slight increase in the high-frequency pre-emphasis (usually by about one $\mathrm{dB}$ at 15 ips) to restore maximum overall flatness in frequency response. On the other hand, the use of the newer tapes on machines designed for earlier types of tapes often results in a considerable degree of excess pre-emphasis at high frequencies, and the effect of overbiasing can be used to compensate for this excess.

\subsection{Noise Balance}

This control is probably the most commonly ignored control on the tape recorder. Some manufacturers recommend setting the control for minimum second-harmonic distortion, or alternatively for minimum noise in the playback output when no signal is recorded. Using the latter technique, the null in playback noise is too broad, and the search for the null is usually inadequate. For really good recordings with a minimum of distortion, the best setting of the control is that which minimizes second-harmonic distortion.

A convenient test frequency for this adjustment is $400 \mathrm{~Hz}$, and it should be set at about 0 vu level on the recording level meter, but these adjustments are not critical. It is important, however, to monitor the distortion with a wave analyzer, and the signal source used for the adjustment must itself be verified to introduce less than 0.1 percent second-harmonic distortion. Otherwise, it is likely that you will introduce into the nulling process a distortion of equal magnitude and opposite 
phase. Unless a laboratory has at hand equipment that permits making this adjustment, it cannot be sure of making recordings of very high quality. Purely by chance, the recorder might arrive from the manufacturer with this adjustment optimized, but it is unwise to count upon the stability of this good fortune. The adjustment must be redone if any of the bias oscillator components, such as tubes or transistors, are replaced.

\subsection{Miscellaneous Good Housekeeping Procedures}

The need for frequent cleaning and demagnetization of heads, guides and capstans should be obvious, but these items are easy to overlook. In particular, a recorder should have these parts demagnetized after it has been transported to a field location, if the requisite $A C$ power is at hand. If heads have residual magnetization, the background noise level will be increased, and the even-order components of nonlinear distortion will be enhanced. (Be sure to demagnetize the heads before adjusting the noise balance control.)

The conventional head demagnetizer may not produce enough field to remove the magnetization found in a tape transport. A portable magnetometer is most suitable for checking this. A useful discussion of this is found in Ref. [7].

Heads, capstans and idlers should be cleaned at frequent intervals, using a cleaning solvent recommended by the manufacturer of the particular tape recorder in use. Cleaning agents are not interchangeable among recorders, because cements used in head assemblies differ. Even a very small particle of oxide on a head can cause a drastic cut in high-frequency response. Small amounts of oxide on the capstan can cause appreciable flutter. Keeping all elements of the tape transport clean is a fundamental necessity for good tape recording.

Ideally, tape should be stored on the takeup reel after recording or playing back. The winding is usually much smoother at normal tape transport speed, and the tension remaining in the tape is more uniform. Also, print-through tends to dissipate after separation of the tape from the adjacent layer. The time required for rewinding allows partial decay of print-through to take place before the material on the tape is played back. 
Print-through increases markedly with increasing temperatures, so that tapes should not be stored for any period of time at temperatures in excess of normal room temperature. A common, and unfortunate, environment to which tapes obtained in the field are exposed with undue frequency is the interior of a closed automobile in the summertime. Good storage conditions for tapes are compatible with comfortable environment for human beings: about $70^{\circ} \mathrm{F}$ and 50 percent relative humidity.

\section{TAPE RECORDERS FOR RESEARCH PURPOSES}

There is no economy in buying a tape recorder that cannot perform as we1l as is needed for the work at hand. By the time you include the cost of a good engineer and the expense of accumulating the sound source material that you are going to record, even the most expensive generalpurpose tape recorders are only a small part of the total budget. (As a matter of fact, you are recording the material for playback precisely because rerunning of the original source of the sound data in order to interpret it is too complicated a process.)

Speed control and uniformity of tape motion are two major difficulties in the execution of all recorder designs. In large console machines the capstan speed usually is maintained by a massive flywheel. Powerful motors and good brakes are essential. Brake assemblies should be made out of solid machined stock where possible. We had a memorable experience with an early model of a large, otherwise excellent console tape recorder, when the brake drums separated where they had been spot-welded to the brakeshaft, engulfing us in a shower of what had been our good data, most of it in little ribbons about an inch or two in length.

As the foregoing episode shows, one should be on the alert for errors of omission in good engineering practice. If your requirements include rapid starting and stopping of the recorder, you must expect to have a tape recorder whose drive motors are powerful, and the mechanical structure holding the tape deck must be correspondingly rugged. For rapid starting and stopping, with rapid attainment of final speed, you can expect that the recorder itself will be relatively heavy, and require a large power supply. 
If you can allow ample time for attainment of final speed, you will find that speed stability can be maintained in a small portable tape recorder within the same tolerances for wow and flutter that one can expect to find in a large studio console. The most successful small recorders use servo feedback to sense and stabilize the capstan speed. Several battery-powered portable recorders use this process for speed stabilization; there are also portable power-line operated tape recorders that make use of servo feedback. A great advantage of this type of speed stabilization in addition to its light weight is that it has much lower inertia than a flywheel, and permits detailed variation in playback speed where necessary.

Most recorders use synchronous motors to maintain the standard speed, and are not suitable for field use powered from a generator or converter unless some very good process of stabilizing line frequency is available. However, the portable recorders that incorporate servo feedback offer the promise of usable speed stability even when operated from an AC converter.

For economy in construction, many home-type tape recorders and even some with modest professional pretensions stabilize the contact between the tape and the heads by means of a spring-loaded pad. This introduces a source of stick-slip friction, with attendant modulation noise. In the tape recorders intended for precise control of recording and playback characteristics, the tension holding the tape against the heads is applied positively via the tape transport. This design works when it is executed properly, but of course it is more complex in structure than a pressure pad, and there are more chances for failure.

Some of the other characteristics which should be expected of a good machine are worth summarizing here. Ideally, the dynamic range should be limited only by the tape medium. The overload levels for the electronic circuits should be substantially above that of the tape, both on recording and playback. The noise should be well below the inherent noise level for the tape. (Some otherwise excellent older machines introduce more noise at low frequencies than does low-noise tape. This noise may be inaudible if one listens to playback, because the ear discriminates against low frequencies to some extent, but still it may be sufficient 
to indicate on flat measuring equipment.) The nolse level should be low enough in the recorder itself to permit the user to take advantage of future improvements in the tape medium. Since the process of tape recording is symmetrical, no even-order distortion should be observed.

The flutter, as measured with the usual 3000-Hz test frequency, and giving weight to components up to as high as $300 \mathrm{~Hz}$, should be held below 0.15 percent. A flutter figure of 0.1 percent or less is frequent in good machines. Although there is no standardized method for measuring flutter components at higher frequencies, a useful qualitative indication can be found by listening to the "noisiness" of a pure tone recorded at a frequency of $5000 \mathrm{~Hz}$ or higher.

Some manufacturers recommend checking for uniformity of drive speed by recording the line frequency on the tape, and then replaying the recording, observing the Lissajous pattern between the line-frequency and the playback by means of an oscilloscope. For a portable recorder, one can use the pure-tone standard frequency broadcasts by radio station WWV on $2.5,5,10,15$ and $20 \mathrm{MHz}$. The signal can be recorded and played back for a Lissajous pattern against the signal received from WW. The broadcasts of WWV are modulated by 440,500 and $600 \mathrm{~Hz}$ in alternate segments. (Schedule information is available from Frequency-Time Broadcast Services Section, National Bureau of Standards, U. S. Department of Commerce, Boulder, Colorado 80302.)

It is advantageous to have the bias frequency well beyond the range of the signals to be recorded. On well-designed machines, the bias frequency will be $100 \mathrm{kHz}$ or higher. If the bias frequency is low, there is a strong probability that it will be recorded as intermodulation with the signal components, and observed as increased noise that is frequency-dependent. (There have been one or two machines with low biasing frequencies that have been remarkably free of this trouble.)

There are instrumentation recorders that may also be used for recording signals in the audiofrequency range. Usually, however, some degree of potential signal-to-noise ratio has been sacrificed to provide special features not relevant to the ordinary audiofrequency range. In particular, the playback head may be designed with a limited number of turns so 
as to have an extended high frequency range. The resultant decreased head output voltage causes the noise produced by the system electronics to limit dynamic range. This effect is acceptable for some kinds of data taking but usually not for audible signals. Generally speaking, where sound recordings of the greatest dynamic range and linearity are needed, they can be obtained with machines designed primarily for audiof requency recording studios.

\section{RERECORDING}

Even in instances where the original signal recorded on a tape has quality acceptable for use, rerecording copies from that original may introduce serious problems. An obvious source of difficulty is the inherent noise of the recording process: as a rule, the tape noise increases by three $d B$ each time the number of tape generations is doubled, e.g., a copy of a copy of a copy of an original tape is three $d B$ noisier than a copy of the original, and $\mathrm{six} d \mathrm{~B}$ noisier than the original. It is not as easy to predict quantitatively the increase in distortion, but it can be expected that distortion will increase with each tape generation. Modulation noise will be increased in a complex fashion; sidebands will be added to earlier sidebands over an ever-spreading range of frequencies. For psychoacoustic work, it is probably unwise to use tapes that are not direct copies of the original material. Where this choice is not available, it may prove worthwhile to use one of the "linearizing" arrangements discussed previously.

Another feature not to be overlooked is the superposition in successive recordings of the deficiencies in the tape machines. If the frequency response is the same for each copying process a peak of perhaps two $\mathrm{dB}$ introduced in the original recording process will have grown to six $d B$ by the third generation - and at this level the peak may be intolerable. The most painstaking care is needed to prevent deviations of this magnitude. An especial problem in this respect is the "head bump", a ripple in the response at the low-frequency end of the spectrum which is a function of the playback-head geometry and the wavelength of the magnetized pattern on the tape. If machines with heads of identical response are used for playing back each generation when making copies, the 
effect will be cumulative. "Head bump" is one of the few phenomena in recording that proves to be less of a problem at $7-1 / 2$ ips than at 15 ips, for it appears at the lower speed for frequencies one octave below where it appears at the higher speed. Phenomena related to head bump and fringing field effects for tracks of different widths may produce unexpected anomalies in the low-frequency response observed when a tape made on one machine is played back on another.

The only safe course for keeping track of recorded data when copying is needed is to record a frequency response and possibly also some tone pairs for distortion checking on the original recording, copying these calibrations along with the program material whenever a dub is made. Where multiple copying techniques are used in the interest of high-speed generation of a number of copies, as in making copies of word lists for distribution, this precaution should always be used to insure that tapes can be checked for uniformity of characteristics. Even with this care being taken, it is risky to attempt high-speed copying processes where accurate work is to be done.

\section{TAPE EDITING}

Often it is necessary to assemble tape-recorded signals in a different sequence, or to change the time-intervals between signals. Tape recordings lend themselves admirably to these rearrangements. It should be obvious that the beginning or end of a desired signal can be found by rocking the tape slowly past the playback head, but evidently this technique has not occurred to everyone: we find a number of word lists in which the tape has been carelessly manipulated, so that words are chopped off near the middle.

Frequent1y it is an aid in editing to inspect the magnetization on the tape by means of a tape viewer. This is a disc upon which a magnetic emulsion is coated, and the particles clump together in the regions of strong magnetic field. The portions of the tape to be edited can be marked with a felt writing pen. Grease pencil, which often is used, leaves undesirable deposits. If the tape cannot be reached when it is in contact with the playback head, a mark can be made at a known distance away from the head, and can be used for a reference. 
For splicing, we have found most convenient those devices which hold the tape in position by means of a slightly tapered groove. The cut is then made with a razor blade. (The blade must be demagnetized before use, or a thump will be developed when the signal is played back in the region occupied by the splice. Sometimes it is necessary to demagnetize the blade repeatedly over several days so that all its residual magnetism is removed before it is used.) The two ends of the tape which are to be joined should be superimposed in the position which they will occupy after splicing, and the cut should be made through both of them in a single operation, so that the cut ends will match precisely. A dull blade will tear and stretch the tape; this is particularly a problem with polyester; it is well to keep a supply of demagnetized cutting blades on hand. The tape viewer is a convenient way of inspecting the cutting blade for residual magnetization.

Ready-made splice sections, which are peeled off a card and then laid on to splice the tape when it is still in position on the cutting block, are the most convenient way of completing the splice. It is difficult to manipulate all parts of the process when the sections of splicing tape must be cut off a roll.

\section{MICROPHONE TECHNIQUES}

In the making of sound recordings in the field, it is important to be aware that the recording-level meter on the tape recorder indicates only the level of signal incident on the recording head. It gives no indication of how much clipping and filtering of the input signal has occurred ahead of that point. In the monitoring of aircraft noise, for example, it is particularly important to ascertain whether any degree of rolling off is occurring in the recording of the low-frequency sounds emitted by the aircraft. With a dynamic microphone, this restriction in frequency range can be introduced by the input coupling transformer. Quite often, the response of microphones intended primarily for the pickup of speech is intentionally rolled off for frequencies below $150 \mathrm{~Hz}$, in order to minimize the pickup of sounds that do not contribute to speech intelligibility. An additional "incentive" for rolling off the low-frequency extreme of the audiofrequency range is the fact that input 
transformers are likely to show saturation at lower signal levels at the low-frequency end of their range than they show at midrange. Since the distortion components are generally of higher frequency than the signal that gave rise to them, they are thrown toward the midrange of the frequencies being recorded, and merely interfere with the dynamic range of signals that can be recorded there.

For these reasons, it is absolutely essential to calibrate the signal directly as it comes off the microphone. A convenient arrangement for doing this also permits the entire recording system to be compensated at a later time for its limitations in frequency range, provided they are not too abrupt. The ground circuit of the microphone is offset from the system ground by means of a resistor whose impedance is low relative to that of the microphone. An auxiliary signal generator is connected through a calibrated attenuator to the resistor, and the voltage output of the generator (usually a sine-wave generator) is monitored at the input to the attenuator. This technique is called an "insert voltage technique". Where maximum precision is desired, each reel of tape has placed upon its input a series of pure-tone recordings with known settings of the signal generator and attenuator, preferably spanning the extremes of the frequency range expected to appear on the recording, and covering a dynamic range extending somewhat above the maximum sound level anticipated. The sound levels which correspond to these signals can be computed from the open-circuit voltage response of the microphone.

This recording of the insert signal must be done with the microphone in a reasonably quiet location, but it has the great advantage that the known signal is introduced into the recording system at precisely the same location as the signal arising from the sound field. Thus the taperecorded signal carries with it at all times the information needed to reduce the data on it to an absolute sound level. An alternative procedure is to record the data through the microphone system of a soundlevel meter of high quality, monitoring the readings of the sound-level meter in terms of all octave band indications available as well as the overall "linear" weighting scale. A sound-level meter conforming to the ANSI standard for precision sound-level meters should be capable of 
linear indication over the range of signals to be recorded. It will then become clear whether the tape recorder itself is being overloaded, if it proves impossible to duplicate these readings, at least in terms of relative levels, upon playback of the tape. This arrangement requires no modification of existing equipment, but is less likely to indicate the sources of nonlinearity than is the insert method.

There is a tendency to use dynamic microphones for making sound recordings in the field because of their apparently rugged structure. However, for measurements of aircraft flyover noise, where the problem is more likely to be very high sound levels and saturation effects, we recommend the use of one-half inch condenser microphones. Whereas it is possible to drop a dynamic microphone and find that it still picks up sound, it is probable that the microphone may have sustained as much damage to its precision of calibration as would a condenser microphone after similar mistreatment.

The relatively large size of dynamic microphones makes orientation a problem, because of the diffraction of high-frequency sounds. On onehalf inch condenser microphones, errors of orientation over the ordinary audiofrequency range likely to be important for airborne sound, i.e. below ten $\mathrm{kHz}$, will not amount to more than a few $\mathrm{dB}$. In addition, such a microphone will be "flat" within $\pm 1 \mathrm{~dB}$ over most of the audiofrequency range, because of its very simple structure and high damping. Very carefully adjusted damping is needed to produce the same degree of "flatness" in a dynamic microphone. The sensitivity of a one-half inch condenser microphone will probably be lower than that of most dynamic microphones intended for the ordinary range of sound levels, but in the case of aircraft flyover noise the problem is not sensitivity, but saturation.

The only drawback to the use of a condenser microphone in field recordings is the relaxation oscillation that may appear when measurements are taken under conditions of high humidity. The breakdown of the polarizing charge gives rise to "pop" sounds, occurring first at infrequent but somewhat regular intervals. Ultimately, the rate of breakdown becomes rapid and the resultant signal seems like random noise. This is easily mitigated by carrying a small sealed box for the microphone, fitted with 
a container of fresh desiccating material, such as silica gel. One-half hour in the drying chamber will permit several hours' use of the microphone before the breakdown problem recurs.

Several manufacturers of sound-level meters provide condenser microphones and preamplifiers with their instruments as optional equipment; they also are available from manufacturers of broadcast equipment, both here and abroad. They are readily adapted for use with an insert calibrating technique, and their high impedance is particularly convenient for this purpose, since it permits the use of ordinary attenuators intended for line use (typically $600 \mathrm{ohms}$ ), and the voltages to be measured are likewise conveniently larger than for the dynamic microphones.

Measurements of sound in open fields - literally in the field - require some arrangement to protect the microphone against wind turbulence. Although it is less of a problem for the stiff-plate end structure that is the diaphragm of a condenser microphone than it is for the compliant diaphragm of a dynamic microphone, it must be dealt with. Commercial wind screens are available.

References [8] and [9] relate to the application of wind screens to microphone measurements in the field. 


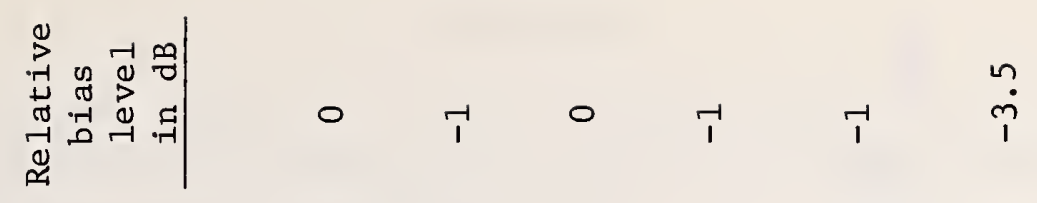

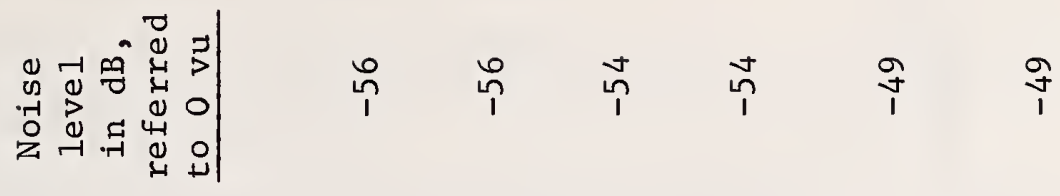

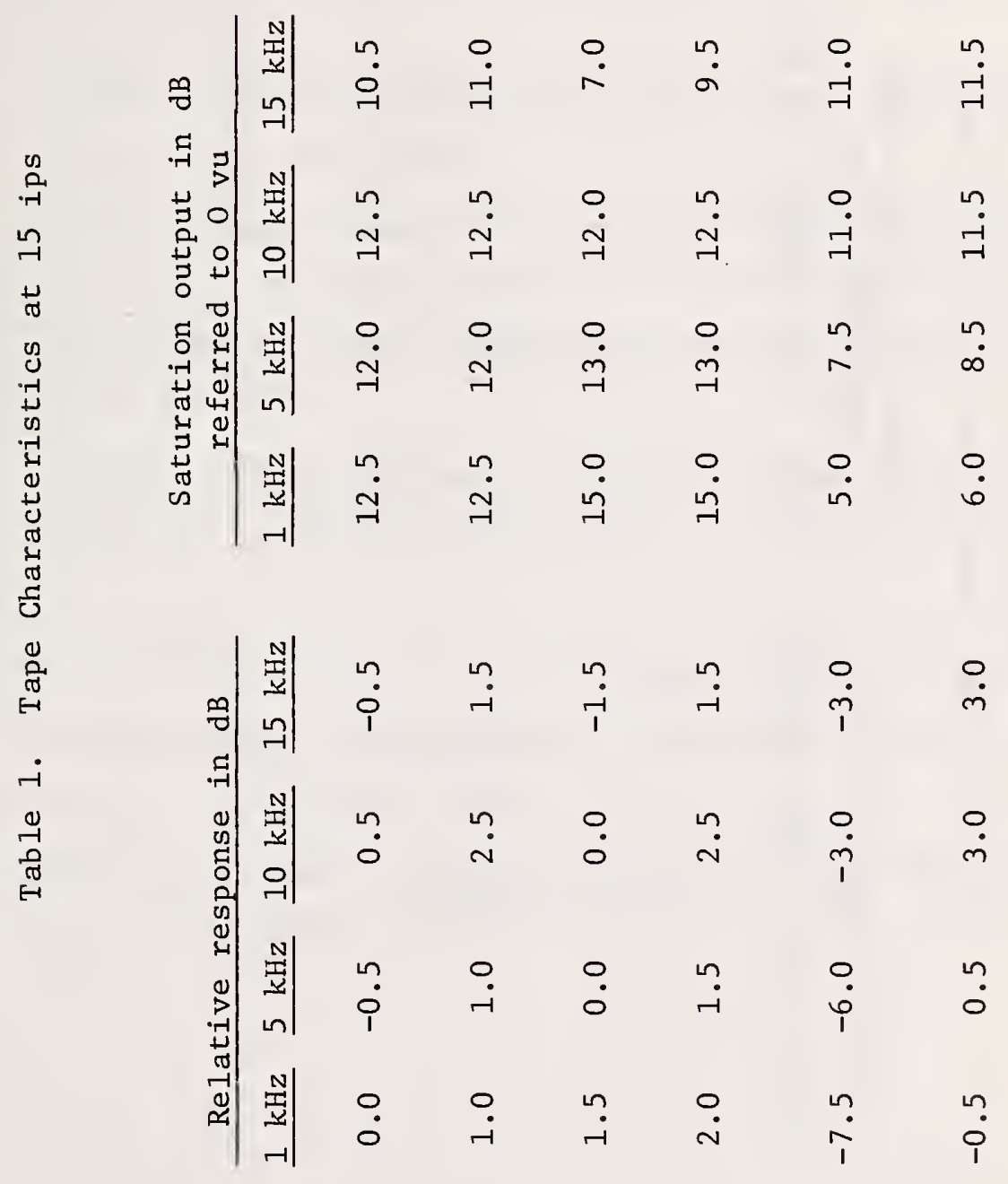

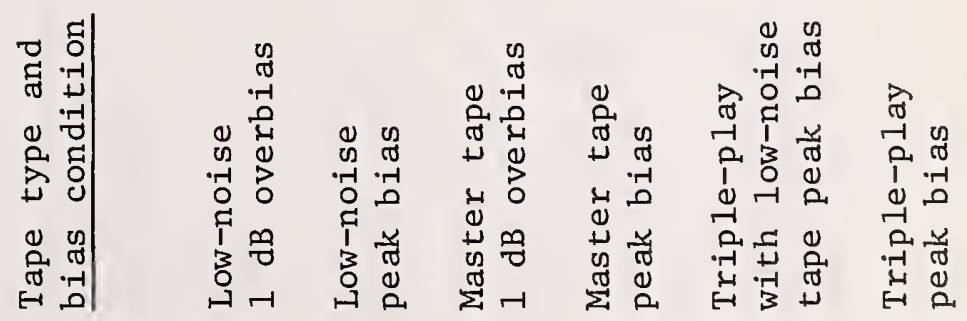




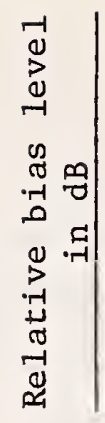

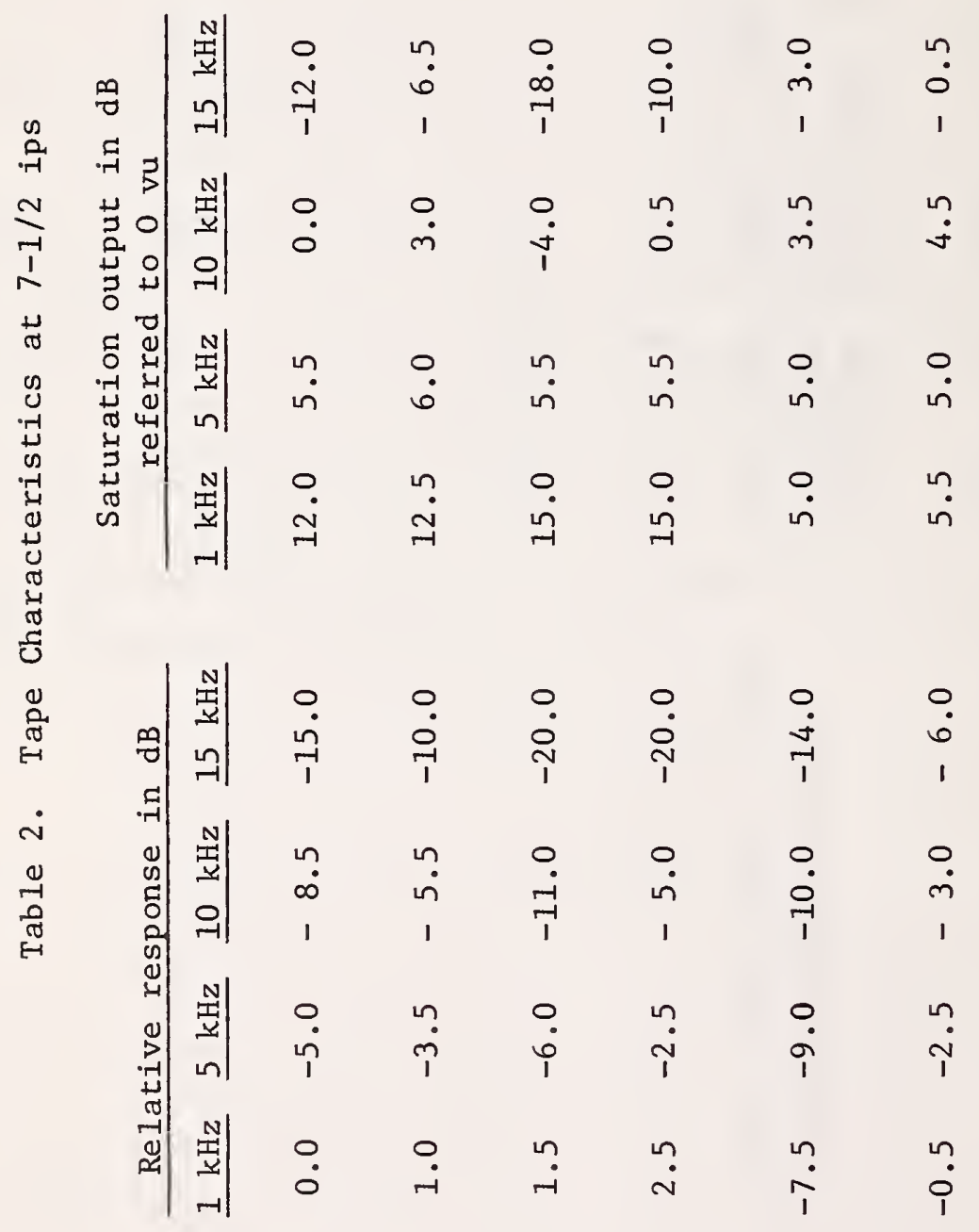

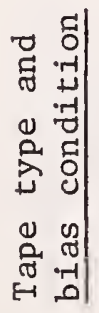

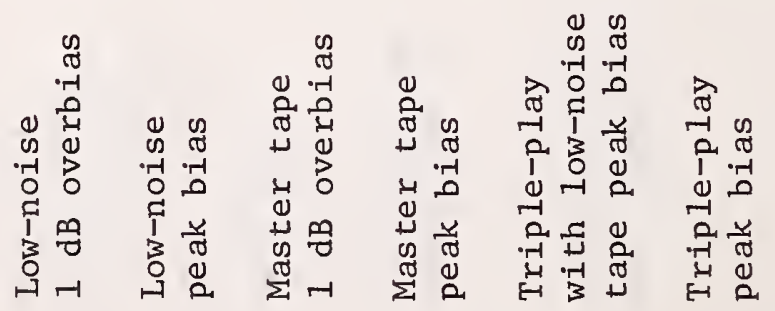


[1] J. G. McKnight, "Absolute Flux and Frequency Response Characteristics in Magnetic Recording: Measurements, Definitions, and Standardization", J. Audio Eng. Soc. 15, 254 (1967).

[2] Angus McKenzie, "Professional Recording Tapes Surveyed", Studio Sound (England) 12, 475 (1970).

[3] R. M. Dolby, "An Audio Noise Reduction System", J. Audio Eng. Soc. 15, 383 (1967).

[4] R. Z. Langevin, "Dynamic Range Limitations in Tape Recording", J. Audio Eng. Soc. 12, 294 (1964).

[5] J. G. McKnight, "The Frequency Spectra of Several Languages as a Criterion for the Frequency Response of Language Laboratories", 15th Annual Meeting of the Audio Engineering Society, Preprint No. 288, October 14-18, 1963.

[6] E. G. Trenda11, "The Measurement and Subjective Assessment of Modulation Noise in Magnetic Recording", J. Audio Eng. Soc. 17, 644 (1969).

[7] Bert Whyte, "Behind the Scenes", Audio Jan. 1971, 10; May 1971, 12.

[8] L. L. Beranek, Acoustic Measurements, John Wiley and Sons, Inc., New York, N. Y., 1949, 258-259.

[9] H. F. Olson and F. Massa, Applied Acoustics, P. Blakiston's Son and Co., Inc., Philadelphia, Pa., 1939, 155. 


\section{GLOSSARY}

Bias - A high frequency sinusoid which is added to, but not modulated by, the audio signal in order to produce a linear amplitude characteristic in the recording.

Dynamic range - The difference in decibels between the noise level of the system and its overload point.

Peak program meter - A meter which, with its associated circuitry, has a very fast rise and slow decay so that the peaks in the program material are indicated.

Print-through - The transferring of signals from a layer of tape to adjacent layers.

Servo feedback - A method of speed control for a mechanism - usually rotating. A common procedure is to place a permanent magnetization pattern on the shaft or use a magnetized gear so that a stationary detecting coil is subjected to a changing magnetic field. The magnitude or frequency of the voltage induced on the pickup coil is used to derive an inverse-feedback signal that controls the current driving the mechanism. Signal-to-noise ratio - The ratio between the magnitude of the signal to that of the noise. In tape recording, a signa1 leve 1 of +6 vu is common $1 y$ used when determining this ratio. A weighted signal-to-noise ratio, especially one using an A-weighting for the noise, may be used for some applications. The characteristics of an A-weighting may be found in ANSI standard S1.4-1971.

VU meter - A meter having ballistic and scale characteristics as specified in ANSI Standard C16.5-1954 (R1961). The term is often carelessly used to indicate any type of monitoring meter on a tape recorder.

Wow and flutter - Deviations from a uniform tape speed. Wow is a slow periodic change in speed, whereas flutter results from an irregular motion of the tape. 


\begin{tabular}{|c|c|c|c|}
\hline \begin{tabular}{c|c} 
U.S. DEPT. OF COMM. & I. PUBLICATION OR REPORT NO. \\
BIBLIOGRAPHIC DATA & NBS -TN -718 \\
\end{tabular} & $\begin{array}{l}\text { 2. Gov't Accession } \\
\text { No. }\end{array}$ & \multicolumn{2}{|c|}{ 3. Recipient's Accession No. } \\
\hline \multirow{2}{*}{\multicolumn{2}{|c|}{$\begin{array}{l}\text { 4. TITLE AND SUBTITLE } \\
\text { Magnetic Recording of Acoustic Data on Audiofrequency } \\
\text { Tape Recorders }\end{array}$}} & \multicolumn{2}{|c|}{$\begin{array}{l}\text { 5. Publication Date } \\
\text { Apri1 } 1972\end{array}$} \\
\hline & & \multicolumn{2}{|c|}{ 6. Performing Organization Code } \\
\hline $\begin{array}{l}\text { 7. AUTHOR(S) } \\
\text { Edwin, D. Burnett, Edith L. R. Corliss, Raymo }\end{array}$ & . Berendt & 8. Perfor & g Organization \\
\hline \multirow{2}{*}{\multicolumn{2}{|c|}{$\begin{array}{l}\text { 9. PERFORMING ORGANIZATION NAME AND ADDRESS } \\
\qquad \begin{array}{l}\text { NATIONAL BUREAU OF STANDARDS } \\
\text { DEPARTMENT OF COMMERCE } \\
\text { WASHINGTON, D.C. } 20234\end{array}\end{array}$}} & \multicolumn{2}{|c|}{$\begin{array}{l}\text { 10. Project/Task/Work Unit No. } \\
2130110\end{array}$} \\
\hline & & \multicolumn{2}{|c|}{ 11. Contract/Grant No. } \\
\hline \multirow{2}{*}{\multicolumn{2}{|c|}{ 12. Sponsoring Organization Name and Address }} & \multicolumn{2}{|c|}{$\begin{array}{l}\text { 13. Type of Report \& Period } \\
\text { Covered } \\
\text { Final }\end{array}$} \\
\hline & & \multicolumn{2}{|c|}{ 14. Sponsoring Agency Code } \\
\hline \multicolumn{4}{|l|}{ 15. SUPPLEMENTARY NOTES } \\
\hline \multicolumn{4}{|c|}{$\begin{array}{l}\text { 16. ABSTRACT (A 200-word or less factual summary of most significant information. If document includes a significant } \\
\text { bibliography or literature survey, mentionit here.) } \\
\text { This Technical Note discusses the application of magnetic tape recording to storage and } \\
\text { analysis of data obtained in the course of acoustic measurements. In general, the most } \\
\text { suitable machines are those designed primarily for recording studios. Advantages in } \\
\text { signal-to-noise ratios and extended linear range can be realized by careful choice of } \\
\text { recording medium, adjustment of the equipment to optimum performance, and proper care } \\
\text { in monitoring the recording process. General instructions for realizing these advantage } \\
\text { are given. Particular emphasis is given to the procedures for making well-calibrated } \\
\text { recordings in the field, for analysis of noise problems. }\end{array}$} \\
\hline \multicolumn{4}{|c|}{$\begin{array}{l}\text { 17. KEY WORDS (Alphabetical order, separated by semicolons) } \\
\text { Acoustic measurements; calibrated tape recordings; data acquisition; } \\
\text { magnetic recording; psychoacoustic data; recordings in the field; tape recording. }\end{array}$} \\
\hline \multicolumn{4}{|c|}{$\begin{array}{l}\text { 18. AVAILABILITY STATEMENT } \\
\begin{array}{l|l}\mathrm{X} \text { UNL IMITED. } & \begin{array}{c}\text { 19. SECURITY CLASS } \\
\text { (THIS REPURT) }\end{array} \\
\text { UNCL ASSIF IED }\end{array}\end{array}$} \\
\hline $\begin{array}{l}\square \text { FOR OFFICIAL DISTRIBUTION. DO NOT RELEASE } \\
\text { TO NTIS. }\end{array}$ & $\begin{array}{l}\text { 20. SECUR I } \\
\text { (THIS P } \\
\text { UNCL A }\end{array}$ & $\begin{array}{l}\text { YCLASS } \\
\text { GE) } \\
\text { IFIED }\end{array}$ & $\begin{array}{l}\text { 22. Price } \\
40 \text { cents }\end{array}$ \\
\hline
\end{tabular}





\section{See How The...}

\section{NATIONAL TECHNICAL INFORMATION SERVICE}

U.S. Department of Commerce can help your firm

- DEVELOP, IMPROVE PRODUCTS

- REDUCE COSTS

- INCREASE PRODUCTION

- PUT NEW DATA INTO YOUR

R\&D PROGRAM AT LOW COST

Get these guides to over 50,000

newly released Government reports each year.

GOVERNMENT REPORTS ANNOUNCEMENTS (GRA).

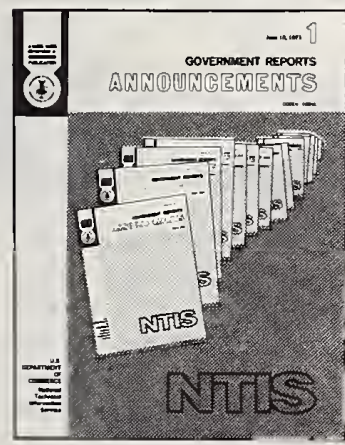

\section{GOVERNMENT REPORTS INDEX.}

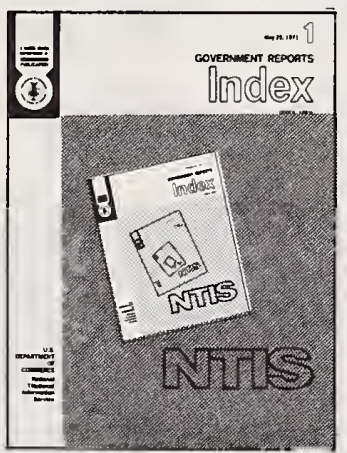

Published concurrently with GRA. Indexes each issue of GRA by subject, personal author, corporate author, contract number, and accession/report number.

Keep up-to-date on current Government R\&D activity.

\section{JUST RETURN THIS HANDY COUPON NOW.}

Mail Now to:

National Technical Information Service

U.S. Department of Commerce Springfield, VA. 22151

$\square$ Begin my subscription immediately to: Government Reports Announcements and its companion volume, Government Reports Index. Combined annual subscription: \$97.50; overseas mail, $\$ 125$.

At this time I wish to subscribe only to:

$\square$ Government Reports Announcements: annual subscription, $\$ 52.50$; overseas mail, $\$ 67.50$.

$\square$ Government Reports Index: annual subscription, $\$ 57.50$; overseas mail, $\$ 67.50$.

$\square$ Bill me (add 50द). $\square$ Here is my check.

$\square$ Charge my NTIS Deposit Account No._.

$\square$ Also send me more information about other NTI5 products and services. __ including the Annual Cumulative Index to GRA.
Name

Organization

Address

City, State, ZIP

Field of Interest

Signed 


\section{If you're looking for a solution to your ADP standards problems ...}

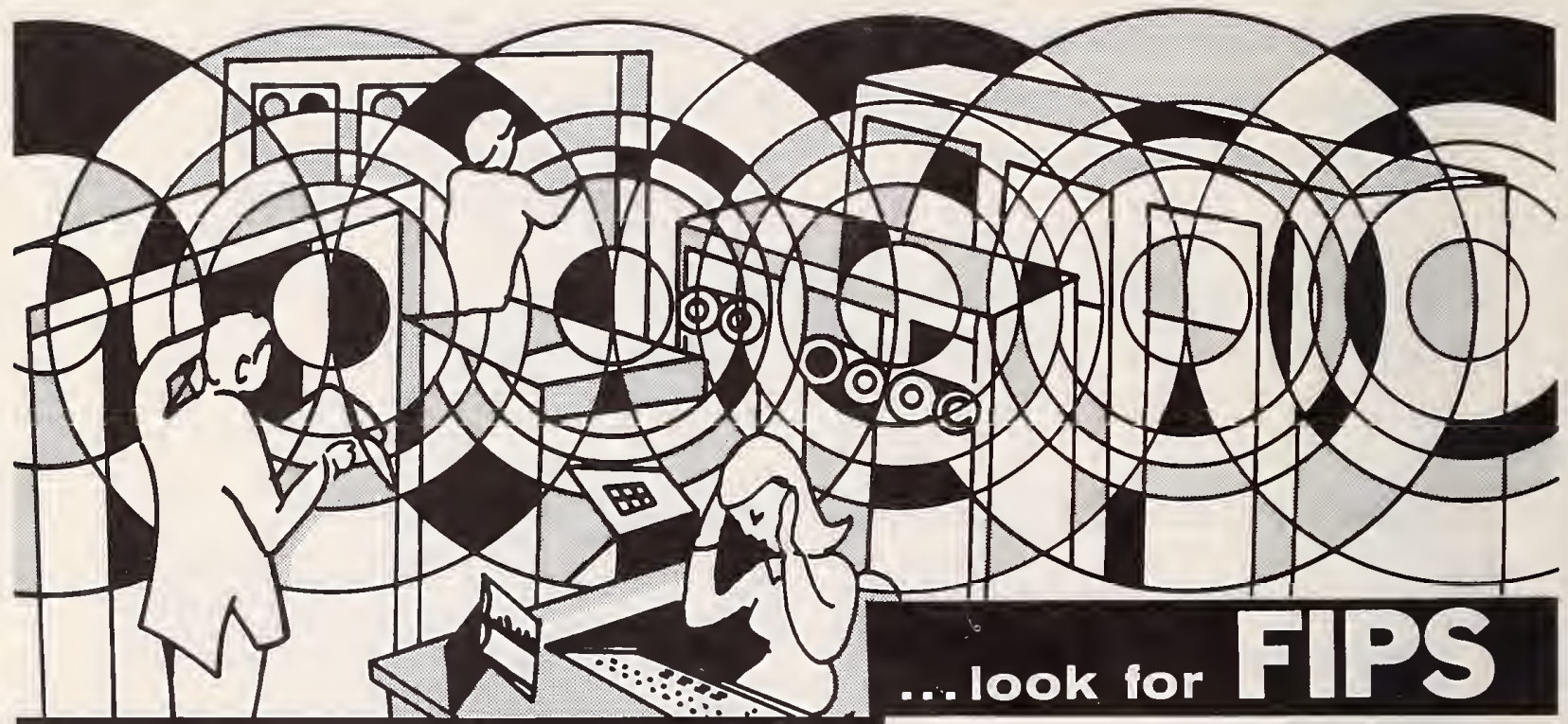

DO YOU need up-to-date information on Federal Stand: ards in the computer/information processing field?

MUST YOU keep abreast of standards adopted under PL 89-306 (Brooks Bill)?

THEN YOU need to subscribe to NBS, FIPS Pub series! (Federal Information Processing Standards Publications Series of the National Bureau of Standards)
FIPS PUBS contain Federal standards for hardware, software, applications and data. FIPS PUBS provide information on new and revised Federal standards as they become available.

FIPS PUBS are the official U.S. Government publications for Federal computer/informa. tion processing standards.

FIPS PUBS ARE AVAILABLE ON A SUBSCRIPTION BASIS FROM THE U.S. GOVERN. MENT PRINTING OFFICE. USE ATTACHED ORDER FORM.

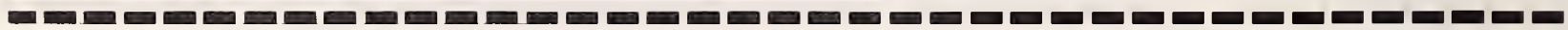

\section{ORDER FORM}

To: Superintendent of Documents, Government Printing Office,

Washington, D. C. 20402

Enclosed find $\$ 12.00$ (check, money order or Supt. of Documents coupons) for a subscription to the National Bureau of Standards Federal Information Processing Standards Series. (For foreign mailing add \$3.00.)

$\square$ Please charge these Name publications to my

Deposit Account No.

Street address

City, State, and ZIP Code

FOR USE OF SUPT. DOCS.
Enclosed
To be mailed
later
Subscription
Refund
Coupon refund
Postage

TO INSURE PROMPT, ACCURATE SHIPMENT, PLEASE PLACE CORRECT ADDRESS ON MAILING LABEL BELOW U.S. GOVERNMENT PRINTING OFFICE DIVISION OF PUBLIC DOCUMENTS WASHINGTON, D. C. 20402 OFFICIAL BUSINESS RETURN AFTER FIVE DAYS

Street address

City, State, and ZIP Code
POSTAGE AND FEES PAID U.S. GOVERNMENT PRINTING OFFICE 
JOURNAL OF RESEARCH reports National Bureau of Standards research and development in physics, mathematics, chemistry, and engineering. Comprehensive scientific papers give complete details of the work, including laboratory data, experimental procedures, and theoretical and mathematical analyses. Illustrated with photographs, drawings, and charts.

Published in two sections, available separately:

\section{- Physics and Chemistry}

Papers of interest primarily to scientists working in these fields. This section covers a broad range of physical and chemical research, with major emphasis on standards of physical measurement, fundamental constants, and properties of matter. Issued six times a year. Annual subscription: Domestic, $\$ 9.50 ; \$ 2.25$ additional for foreign mailing.

\section{- Mathematical Sciences}

Studies and compilations designed mainly for the mathematician and theoretical physicist. Topics in mathematical statistics, theory of experiment design, numerical analysis, theoretical physics and chemisty, logical design and programming of computers and computer systems. Short numerical tables. Issued quarterly. Annual subscription: Domestic, \$5.00; $\$ 1.25$ additional for foreign mailing.

\section{TECHNICAL NEWS BULLETIN}

The best single source of information concerning the Bureau's research, developmental, cooperative, and publication activities, this monthly publication is designed for the industry-oriented individual whose daily work involves intimate contact with science and technology-for engineers, chemists, physicists, research managers, product-development managers, and company executives. Annual subscription: Domestic, $\$ 3.00 ; \$ 1.00$ additional for foreign mailing.
Applied Mathematics Series. Mathematical tables, manuals, and studies.

Building Science Series. Research results, test methods, and performance criteria of building materials, components, systems, and structures.

Handbooks. Recommended codes of engineering and industrial practice (including safety codes) developed in cooperation with interested industries, professional organizations, and regulatory bodies.

Special Publications. Proceedings of NBS conferences, bibliographies, annual reports, wall charts, pamphlets, etc.

Monographs. Major contributions to the technical literature on various subjects related to the Bureau's scientific and technical activities.

\section{National Standard Reference Data Series.}

NSRDS provides quantitative data on the physical and chemical properties of materials, compiled from the world's literature and critically evaluated.

Product Standards. Provide requirements for sizes, types, quality, and methods for testing various industrial products. These standards are developed cooperatively with interested Government and industry groups and provide the basis for common understanding of product characteristics for both buyers and sellers. Their use is voluntary.

Technical Notes. This series consists of communications and reports (covering both other agency and NBS-sponsored work) of limited or transitory interest.

Federal Information Processing Standards Publications. This series is the official publication within the Federal Government for information on standards adopted and promulgated under the Public Law 89-306, and Bureau of the Budget Circular A-86 entitled, Standardization of Data Elements and Codes in Data Systems.

Consumer Information Series. Practical information, based on NBS research and experience, covering areas of interest to the consumer. Easily understandable language and illustrations provide useful background knowledge for shopping in today's technological marketplace.

NBS Special Publication 305, Supplement 1, Publications of the NBS, 1968-1969. When ordering, include Catalog No. C13.10:305. Price \$4.50; $\$ 1.25$ additional for foreign mailing.
Order NBS publications from:
Superintendent of Documents

Government Printing Office

Washington, D.C. 20402 
PDSTAGE AND FEES PAID

JFFICIAL BUSINESS

Jenalty for Private Use, $\$ 300$

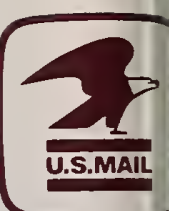

